\title{
Dynamic Transverse Debonding of a Single Fiber
}

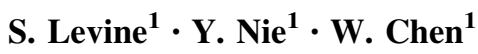

Received: 25 August 2016/ Accepted: 24 October 2016/Published online: 28 October 2016

(C) Society for Experimental Mechanics, Inc 2016

\begin{abstract}
By integrating a Kolsky tension bar along with a high-speed x-ray phase contrast imaging method and a high speed optical camera, high strain rate debonding processes of an opaque composite composed of a single S-2 fiber and SC15 epoxy resin is recorded in real time and analyzed. Specifically, imaging of transverse debonding initiation and progression along with debonding loads are obtained and then compared to quasi-static debonding loads in this research. The dynamic loading direction was perpendicular to the fiber axial direction. The dynamic tensile loading history as the debonding initiated and propagated was recorded using a piezoelectric load cell. The initial debonding crack speed was also measured. Quasi-static experiments were also completed as a basis of comparison. It is shown that the debonding loads under dynamic and quasistatic loading conditions are similar. The fracture surfaces from the dynamically debonded samples were subsequently examined by scanning electron microscopy.
\end{abstract}

Keywords Kolsky bar · Single fiber debonding · Synchrotron X-ray $\cdot$ High speed imaging

\section{Introduction}

\section{Transverse Debonding}

Fiber reinforced composites are becoming increasingly common due to their high strength to weight ratios as

W. Chen

wchen@purdue.edu

1 AAE and MSE, Purdue University, West Lafayette, IN 47907, USA compared to more conventional materials. Along with this increased use comes the need to have a higher level of understanding of the material characteristics. Specifically, the interface between the fiber and matrix is of interest. Loss of adhesion at this interface, known as debonding, can greatly decrease material strength. There has been significant research into debonding phenomena at low strain rates. However, there is still a need for further insight at higher strain rates. In addition, given the opacity of many epoxy resins, conventional imaging is often unable to record debonding events or is restricted to only transparent matrices.

Transverse debonding experiments were developed because it was often observed that the initial debonding failure mode in fiber reinforced composites was in the transverse direction [1]. Keeping with the trend used with previously developed experiments, transverse debonding experiments were often conducted with a single fiber as opposed to multiple fibers. This was due to the fact that it was much easier to analyze the failure of a single fiber. With a group of fibers, debonding of one fiber would affect the strength characteristics of neighboring fibers. By using a single fiber sample, this complexity was eliminated [2]. Also, a single fiber sample was much quicker and cost effective to manufacture [2]. While a single fiber sample is not as representative of a true fiber reinforced composite, it does allow for much easier characterization and visualization of debonding events, as well as providing the fundamental debonding information.

The main factor that drove specimen geometry for transverse debonding experiments was the free edge effect. This property was first analyzed by Pipes and Pagano [3]. The free edge effect is a stress singularity that exists at the point where a fiber meets the free surface in a fiber reinforced composite [4]. In a transverse debonding experiment 
with a single fiber, analytical and numerical analyses predict that the stress singularity is sufficient to create interfacial debonding at the free surface before any load is applied. Further evidence showed that premature debonding occurred at the fiber/matrix interface at the free surface [4]. While this is truly indicative of behavior at the edges of fiber reinforced composite structures, this is not always a desired result as it is not representative of fiber/matrix debonding in the middle of a composite structure. Therefore, it was of interest to isolate the free edges from the applied load [4]. Furthermore, having the debonding initiate in the middle of the specimen provided predictable and repeatable experimental results. Given this, different methods had been created in order to characterize transverse debonding while attempting to minimize the influence of the free edge effect. One of the first experiments developed that drastically reduced the free edge effect was the single fiber Broutman test. Specimens for this experiment had a necked design and therefore debonding initiated in the middle of a sample as opposed to at the ends [5]. Although this result was preferred as it eliminated debonding initiating at a free surface, it still had several drawbacks. Firstly, the stress in the transverse direction was not axisymmetric This made the stress much more difficult to analyze [6]. In addition, there were instances where the fiber would break from the applied compressive stress before the tensile stress was able to create debonding. This result reflected the shear properties of the fiber/matrix interface more than the transverse properties [5]. Therefore, the interface needed to be weak in order to have proper transverse debonding [7]. Given these limitations, new sample geometries and experimental setups were desired. One of the latest configurations used for transverse debonding experiments was the cruciform geometry, developed by Gundel et al. [4]. Cruciform specimens were cross shaped with a fiber laid vertically along the center. The cruciform had the major advantage over rectangular samples in that it did not have a large stress concentration at the free edges. This avoided the issue of attempting to analyze the complex stresses present at the free surface. When a transverse load was applied to these samples, the highest load concentration occurred in the middle of the sample. This then caused the samples to debond in the middle, which was more desirable [4]. The cruciform geometry was further enhanced by modifying the corner radii in order to decrease the stress concentrations in the interior corners. The fillet radius was varied by Tandon, Kim, and Bechel [8] until an optimum radius was found. Before the radius was optimized, it was found that finite element analyses as well as physical experiments showed that the specimens would often fail at the fillet as opposed to at the fiber/matrix interface as desired [8]. Further improvements were made to the cruciform design by performing several parametric studies. The first parametric study was performed by Tandon, Bechel, and Kim [9]. This study was initially conceived because even with the enhanced fillet radius, there were still many cases where it was seen that the sample failed at the fillet instead of the fiber/matrix interface. The geometry was considered optimal when, for a given loading condition, the ratio of radial stress at the fiber to the far field stress was highest. An ideal geometry was then found where the wing height was significantly less than the loading arm width [9]. Bechel and Tandon then performed further experiments with various different materials. They found that the previous optimization study worked best for fibers which were very stiff in the transverse direction. Furthermore, the fibers in the previous cruciform studies were over $100 \mu \mathrm{m}$ in diameter. They wanted to replicate these results with a small diameter graphite fiber, which had a lower transverse stiffness. It was found that the samples with the new fiber did not have the tendency to debond and fail at the interface. Thus, further sample modifications had to be made. A parametric study was again performed by modifying the fiber diameter, sample thickness, and fillet radius. Even after performing this study, the samples still failed at the fillet because the fiber deformed easily. This deformation required a larger load to be applied in order to attempt to debond the fiber. However, this increased load eventually caused failure at the fillet before there was a failure at the interface. The solution to this issue was to add a face sheet to the sample after its initial processing in order to increase the thickness in the fillet region. The face sheets were made from a glass epoxy and provided additional reinforcement to the fillets. The face sheets were rounded so that the largest stress concentration would be as close to the middle as possible. Samples were then able to successfully debond at the fiber/matrix interface. However, ultimate failure still routinely occurred at the edge of the face sheet [10]. Further experimental work was completed by $\mathrm{Li}$ et al. [11]. The novel aspect of this experiment was that transverse debonding tests using a cruciform were completed and the normal and tangential stresses at the interface were determined using finite element models [11]. While the aforementioned advancements were a large leap forward in debonding characterization, there was still room for improvement as nearly all the debonding experiments were visually recorded using a microscope and had been completed under quasi-static loading conditions.

\section{Experiment Visualization Methods}

The majority of single fiber debonding experiments previously discussed had visually captured debonding using a microscope. Furthermore, due to equipment setup, samples in some of these examples could be visually analyzed under a 
microscope only after the experiments had been completed. In addition, large diameter fibers were primarily used in these kinds of experiments as they were easier observe [12]. A novel method that was used to visualize debonding was 3D $\mathrm{X}$-ray microtomography [13]. Using synchrotron X-rays, 3D images of debonding evolution was able to be generated. A synchrotron works by accelerating electrons to near the speed of light. The electrons are then inserted into a circular storage ring. Devices called undulators or wigglers, located at various positions along the storage ring excite the electrons. When the electrons are excited, they give off energy in the form of photons in the X-ray spectrum. The photons then leave the storage ring tangentially. Experiment hutches are set up along these tangential paths in order to allow the $\mathrm{X}$-rays to pass through samples. Once the $\mathrm{X}$-rays pass through a sample, it goes through a scintillator which converts it to visible light. The light is then directed towards a camera. Even though in this experiment the samples were not cruciform shaped and debonding did initiate at the free surface, this experimental method still provided an enhanced way to visualize debonding. By using X-rays, the sample did not need to be transparent and thus many more kinds of epoxy resins could be used. While all of these types of experiments were beneficial for understanding debonding under quasi-static loading, it was still desired to have a higher level of understanding of the fiber/matrix interface under dynamic loading conditions. Therefore, it is desired to develop an experimental technique that can visualize the debonding process in real time under dynamic loading.

\section{Kolsky Bar}

Before discussing dynamic experimental methods, it is necessary to understand the motivation for performing high strain rate experiments. Most material properties that are readily available were determined through quasi-static testing [14]. In order to properly design and manufacture materials that will be used in dynamic environments, one must understand the behavior of those materials under dynamic loading conditions. Given that materials may behave very differently under quasistatic and dynamic loading conditions, the necessity of performing dynamic experiments was made very apparent. One of the most widely used devices for testing materials under dynamic loading conditions is the split Hopkinson pressure bar, also known as a Kolsky bar [14]. A Kolsky tension bar consists of a loading device, an incident bar, a specimen, and a transmission bar.

A buildup of pressure in an air storage tank is suddenly released, which causes a striker, which rides on the incident bar itself to impact a flange at the end of the incident bar. A tensile stress wave travels down the incident bar. The stress wave in the bar travels at the speed of sound within the bar, which is calculated as
$c=\sqrt{\frac{\rho}{E}}$

where $\rho$ and $E$ are the density and modulus of elasticity of the bar respectively. Strain gages mounted on the incident bar measure the strain induced on the bar by the passing wave. The signals from the strain gages are sent to an oscilloscope to be recorded. From the outputted strain $(\varepsilon)$, the velocity of individual particles (v) within the bar can be determined.

$v=\frac{\rho c}{E \varepsilon}$

The Kolsky tension bar is able to create a stress wave that is nearly square and has a constant amplitude [15]. The resulting incident and reflected stress waves are captured using the strain gage adhered to the incident bar [15]. By adding a thin piece of soft paper to the flange, the high frequency waves generated at impact are nearly eliminated [15]. A tensile stress wave is unable to travel through an interface of two objects that are only in contact. In order to compensate for this, the incident and transmission bars often have female threads tapped in them. The sample would then have male threads that when threaded into the female threads allow for the tensile wave to pass through. With the use of a Kolsky bar, a multitude of dynamic loading experiments were able to be performed.

\section{Dynamic Debonding Experiments}

One of the first single fiber debonding experiments that was carried out under dynamic loading conditions was single fiber push-out testing using a Kolsky compression bar by Li et al. [16]. A Kolsky compression bar works in a very similar manner to a tension bar [14]. In this experiment, a tapered punch was connected to the incident bar at the end closest to the sample. As was done in prior fiber pushout testing, a single fiber was embedded in a matrix. This matrix was connected to a support, which was attached to the transmission bar. When the compression wave traveled down the punch, it forced the fiber to debond and move forward until it completely separated from the matrix and fell into the larger cavity of the support [16]. This presented a large step forward in single fiber debonding experiments as it allowed for this phenomenon to occur under dynamic loading conditions. While there was valuable data that was able to be analyzed, there was still a gap as images were not recorded. A technique combining the ability to dynamically debond a sample using a Kolsky bar along with the use of imaging was later developed by Hudspeth et al. [17]. This experimental method incorporated the use of synchrotron X-Rays, which was the technology used for the microtomography experiments, with the dynamic loading abilities of a Kolsky bar. Given that a 
Kolsky bar experiment lasts on the order of $100 \mu$ s, it was necessary to provide high speed imaging that could clearly capture the events that transpired [17]. For this experiment, the X-rays were of very high intensity and thus the scintillator could only be exposed to them for very short periods of time. In order to do that, shutters were employed to block the X-rays and then let them through only at a strategically determined time [17]. It was of critical importance that all timing be very precise in order to make sure the experiment was captured without damaging any of the equipment [17]. The specific experiments that were completed using synchrotron X-rays with the Kolsky bar were single fiber pull-out experiments. In this case a tension bar was used. Due to space restrictions, a load cell was used in place of a transmission bar. This version of the Kolsky bar gave similar results to a full Kolsky bar since the vast majority of the incident wave would not have traveled through the sample to the transmission bar [17]. A device connected to the load cell held a droplet of matrix in place. A single fiber was partially embedded in the matrix and was connected to the incident bar. When the dynamic load was applied, the fiber moved along with the movement of the incident bar and away from the load cell [17]. This represented the first time that dynamic debonding was able to be successfully captured visually, in real time. Even though considerable progress has been made with single fiber debonding experiments, there is still plenty of room for further knowledge on the topic through experiments. One such example is dynamic debonding with a tensile load applied perpendicular to the fiber being synchronized with high speed X-ray imaging. This is the focus of this paper.

\section{Experimental Setup}

\section{Sample Preparation}

For this research project, samples were created by pouring epoxy resin into silicone molds. In order to make the properly dimensioned silicone molds, a $6^{\prime \prime} \times 12^{\prime \prime} \times .5^{\prime \prime}$ aluminum bar was machined to the specifications of a drawing, as shown in Fig. 1. OOMOO ${ }^{\circledR} 30$ pourable silicone rubber provided by Smooth-On was then mixed and poured into the cavity of the aluminum bar. The silicone cured at room temperature for $6 \mathrm{~h}$ and was then removed. The aluminum block was machined so that when the cured silicone was removed from the cavity, it possessed the geometry necessary to make samples, as shown in Fig. 2. In order to maximize the number of samples that could be made at a time, the aluminum block was machined so that the silicone mold contained a cavity for 10 samples that would be connected together when the epoxy resin cured

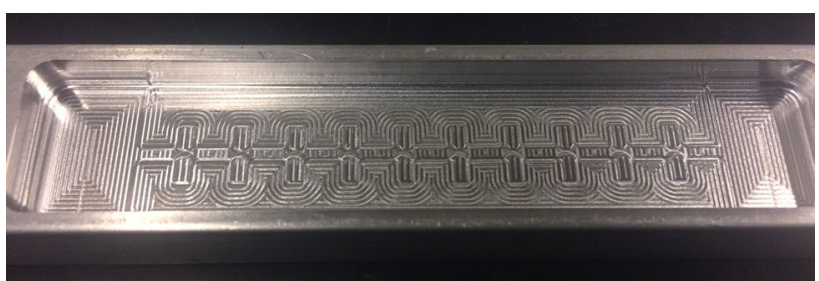

Fig. 1 Machined aluminum block for creating silicone mold

and could then be later separated. After being removed from the aluminum mold, the ends of the silicone mold were scraped using a pair of tweezers in order to make the surface rougher. Super glue would later be applied to the silicone in order to keep the fiber in place. However, given that silicone has very low surface energy, super glue would normally not adhere well to the mold. Therefore, scraping the ends allowed for the super glue to stick to the silicone. The mold was then washed to remove any residue and subsequently dried. Given that the silicone mold was thin, it was very susceptible to bending. Bending proved to have adverse effects on the sample making process, such as possible breakage of cured samples as well as undesired distribution of epoxy resin. In order to compensate for this, the silicone mold was placed on top of an aluminum block, which allowed it to have the necessary rigidity to be handled without bending. A length of a single S-2 glass fiber was then separated from a spool and taped on either end to two additional silicone pieces that were on top of their own aluminum blocks. The S-2 glass fiber, provided by Owens Corning was approximately $10 \mu \mathrm{m}$ in diameter and had a density of $2.46 \mathrm{~g} / \mathrm{cm}^{3}$. By utilizing the two additional silicone pieces on top of their own aluminum blocks, the fiber position could easily be adjusted as needed and could be kept taut by pulling one block away from the other. Furthermore, doing this kept the fiber at the same height from end to end and decreased the chances of it breaking during setup.

The fiber, which was still taped on both ends, was then placed on top of the mold. The aluminum blocks were then adjusted so that the fiber laid along the middle of center channel of the mold. Super glue was applied to the fiber on either end of the mold and a drop of accelerant was placed on the super glue in order to have it harden quicker. The fiber was cut just outside the mold on either end. The next step was to mix and pour the epoxy resin. SC-15 epoxy resin provided by Applied Poleramic was used for the experiments. The epoxy resin was mixed in a beaker in approximately a 100:30 ratio of resin to hardener by weight. After mixing, the beaker was placed in a degassing chamber for $25 \mathrm{~min}$ at $28.5 \mathrm{inHg}$ of vacuum. This allowed for the removal of the majority of the air bubbles within the 
Fig. 2 Cured silicone mold

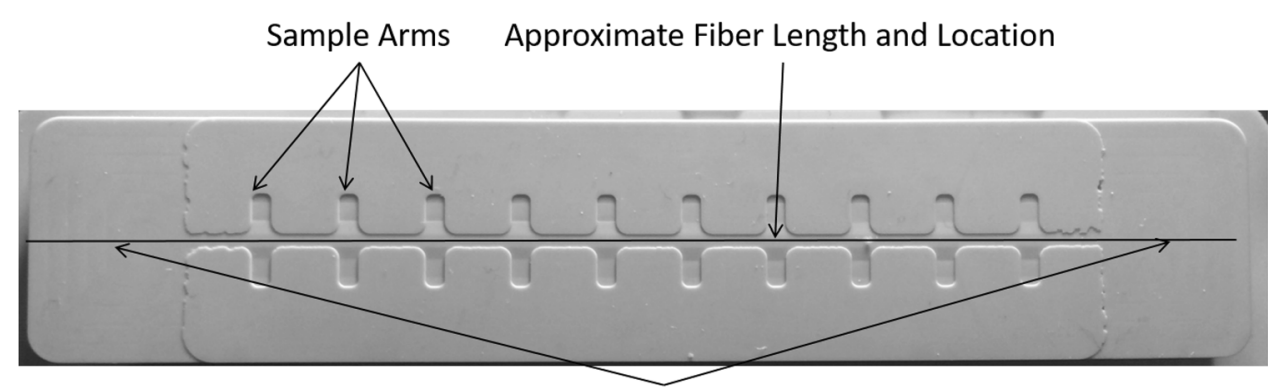

Fiber Adhered to Silicone Here

mixture. After this, a small quantity of the epoxy resin was poured on top of the mold so that all of the features were covered. The mold was then placed in the degassing chamber at $28.5 \mathrm{in} \mathrm{Hg}$ of vacuum for an additional $15 \mathrm{~min}$ to remove any additional air bubbles that formed during the pouring process. A second piece of silicone was placed on the top of the mold with the flat side facing down in order to remove all epoxy resin on the uppermost surface of the mold and to ensure only the epoxy that was in the cavity would remain. A $175 \mathrm{~g}$ aluminum block was placed on top of the flat silicone piece in order to ensure that it pressed down sufficiently to remove excess epoxy resin and that there were no gaps between the two pieces of silicone. This configuration of silicone, aluminum bars, and epoxy was left for 12-16 $\mathrm{h}$ at room temperature so that the epoxy resin could cure. At the end of this time period, the top aluminum block and silicone cover were removed. The samples were then carefully pried out of the mold. While there was some excess epoxy on the mold, it did not connect to the samples and the intended geometry was able to be maintained. The samples were then separated from each other using a scalpel. At this point, the samples were still malleable and could not be used for experiments. Consequently, the samples were left out for another 16-24 h to allow for additional curing. The additional cure time gave the samples sufficient stiffness to be properly tested on the Kolsky bar.

\section{Kolsky Bar Integration}

In order to create dynamic loading conditions, a Kolsky bar was utilized to perform all experiments. The mechanics of the Kolsky bar has been described in "Introduction" section. Grips were designed in order to clamp the samples on either end, as shown in Fig. 3. Each set of grips had a female thread tapped in the back. A set screw was then used to connect one grip to the incident bar and the other grip was secured to a load cell. The load cell had female threads in both the front and back so that it could then be connected to a sample rotator via a set screw.

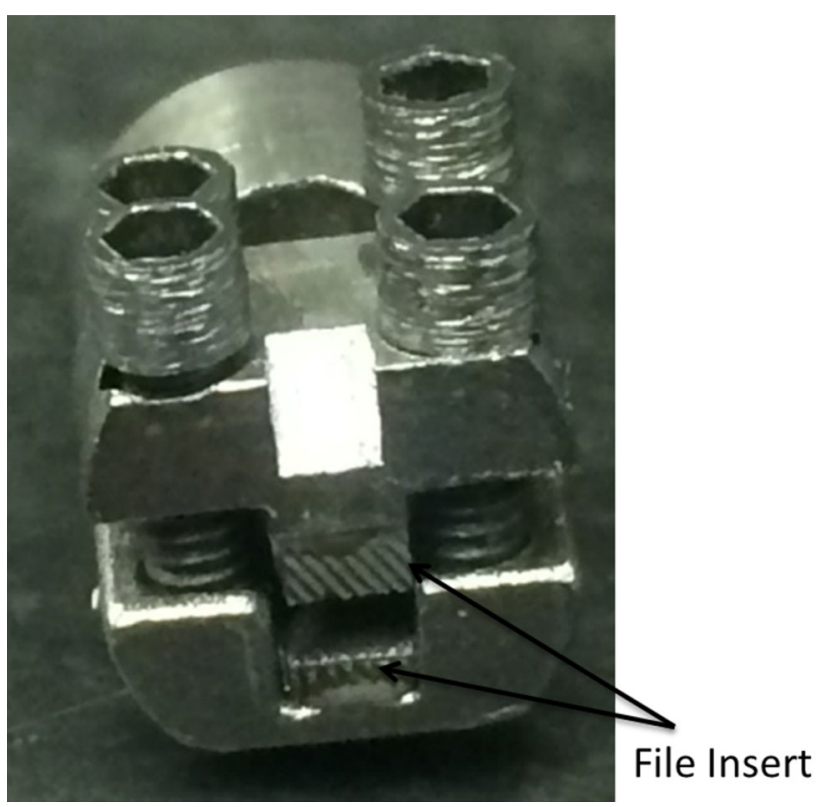

Fig. 3 Opened grip used for tension testing

\section{X-Ray Imaging}

Cured SC-15 epoxy resin is white and very opaque. Given that the fiber was embedded in the sample, a regular optical camera would have been unable to view the debonding initiation. For this reason, high brilliance phase contrast X-ray imaging was utilized in order to properly record images of the debonding initiation and progression. X-ray phase contrast imaging was necessary as it allowed to see through the opaque epoxy resin and also was able to clearly differentiate the fiber from the matrix.

Beamline 32-ID-B at the Advanced Photon Source at Argonne National Lab was utilized in order to provide the $\mathrm{X}$-rays needed for imaging. The experiment hutch had size limitations, so a full Kolsky bar setup with incident and transmission bars could not be fit inside. Therefore, the transmission bar was removed and replaced with a 50-lb load cell provided by Kistler, as shown in Fig. 4. The load cell was able to record at a frequency of $50 \mathrm{MHz}$. The $\mathrm{X}$-ray window was approximately $1 \mathrm{~mm}^{2}$ in size. Therefore, it was important to ensure that the sample was in the correct location when the 
Fig. 4 Test setup on Beamline 32-ID-B

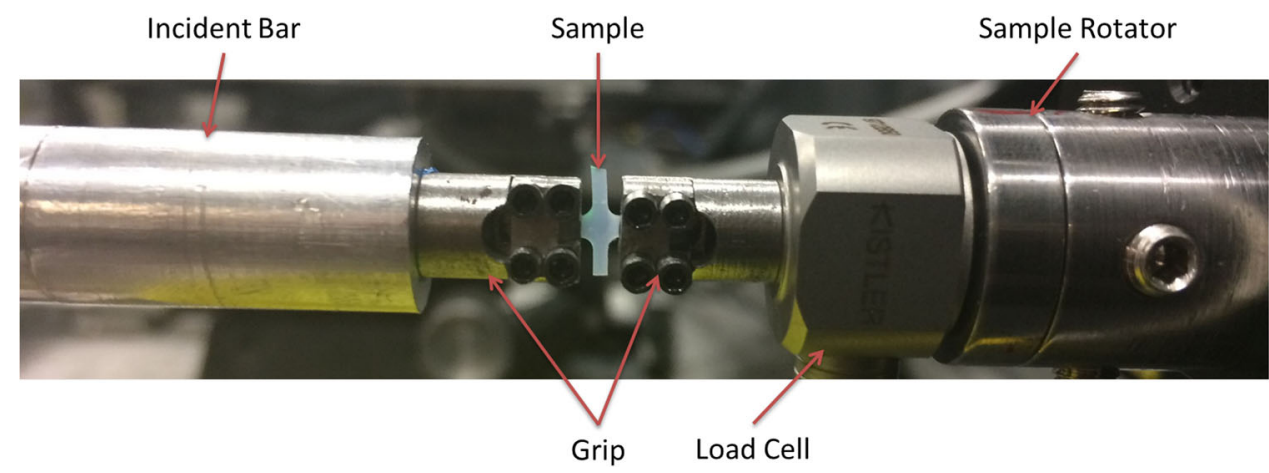

debonding occurred. The sample was located by triggering the camera and momentarily opening the shutters to allow the sample to be exposed to the X-ray beam for a short duration of time. The images were then evaluated in a control room adjacent to the hutch. The Kolsky bar was on a table that was able to move vertically as well as in the direction parallel to the length of the bar using a series of actuators. The actuators could be adjusted from the control room and further images could be taken until the sample was properly aligned with the beam. Once the bar and sample were setup in the proper location, the next important step was to ensure all timing was correct. With all timing verified, proper experimentation and data reduction was able to be performed.

\section{Data Reduction}

In order to properly capture data in the tension experiments, the Kolsky bar was set up as shown in Fig. 5. By utilizing a Kolsky tension bar the samples reached dynamic equilibrium. Therefore, the dynamic experiments are very similar to the quasi-static experiments previously stated except for the loading rates. The first step was to balance the Wheatstone bridge that the strain gauges were connected to in order to ensure an accurate output. The $50 \mathrm{lb}$ Kitsler load cell had a sensitivity of $91.76 \mathrm{lb} / \mathrm{V}$. This value was entered into the amplifier used to output the signal to the oscilloscope. By setting the output for the load cell, the data that was received by the amplifier was then able to be converted to an output voltage. This output voltage was then easily converted into a force based on the amplifier setting. In addition, thread tape was added to the set screw connecting the grip to the incident bar in order to keep the reflected signal as smooth as possible.

The output of the strain gauge and load cell were used to calculate the strain $\left(\varepsilon_{s}\right)$ and displacement of the sample. The strain was:

$\varepsilon_{s}=\frac{2 s}{G F * E V}$

where $s$ was the value outputted from the strain gage, $G F$ was the strain gage factor, and $E V$ was the excitation voltage of the strain gage. For the semi-conductor strain gages used for these experiments, $G F$ was 169 and $E V$ was $28 \mathrm{~V}$. In order to calculate the displacement, the incident particle velocity $\left(v_{I}\right)$ and the reflected particle velocity $\left(v_{R}\right)$ had to be calculated, by utilizing the wave speed of the bar $(c)$. In this case, the transmitted particle velocity $\left(v_{T}\right)$ is assumed to be zero due to the high impedance mismatch between the sample and the load cell.

$V_{I}=c E_{I}$

$V_{R}=c E_{R}$

The sample velocity was found by lining up the incident and reflected waves and adding the reflected velocity to the incident velocity. This was then numerically integrated to give the sample displacement.

\section{Sample Design}

While experiments performed by Tandon and Kim all took place in the quasi-static regime [8], the insight gained from their design modifications was still useful for samples studied under dynamic loading conditions. In these cases, the samples often failed at the interior corners. In order to increase the strength of the corners, a face sheet was added in order to increase the cross sectional area [8]. A similar approach was taken with the samples for this research project, except that instead of adding a separate component for the face sheet, the sample was reinforced with additional epoxy from the edge of the loading arm to the interior corners. This was done by making the mold deeper in this area and thus there was more epoxy in this location. This way, an additional material did not have to be added to the samples. The samples were given a tapered, reinforced loading arm in order to avoid a large stress concentration. By designing the sample thickness in the interface region to be $76 \mu \mathrm{m}$, the ratio of the fiber diameter to sample thickness was maximized, which allowed for a higher likelihood of failure occurring at the fiber/matrix 


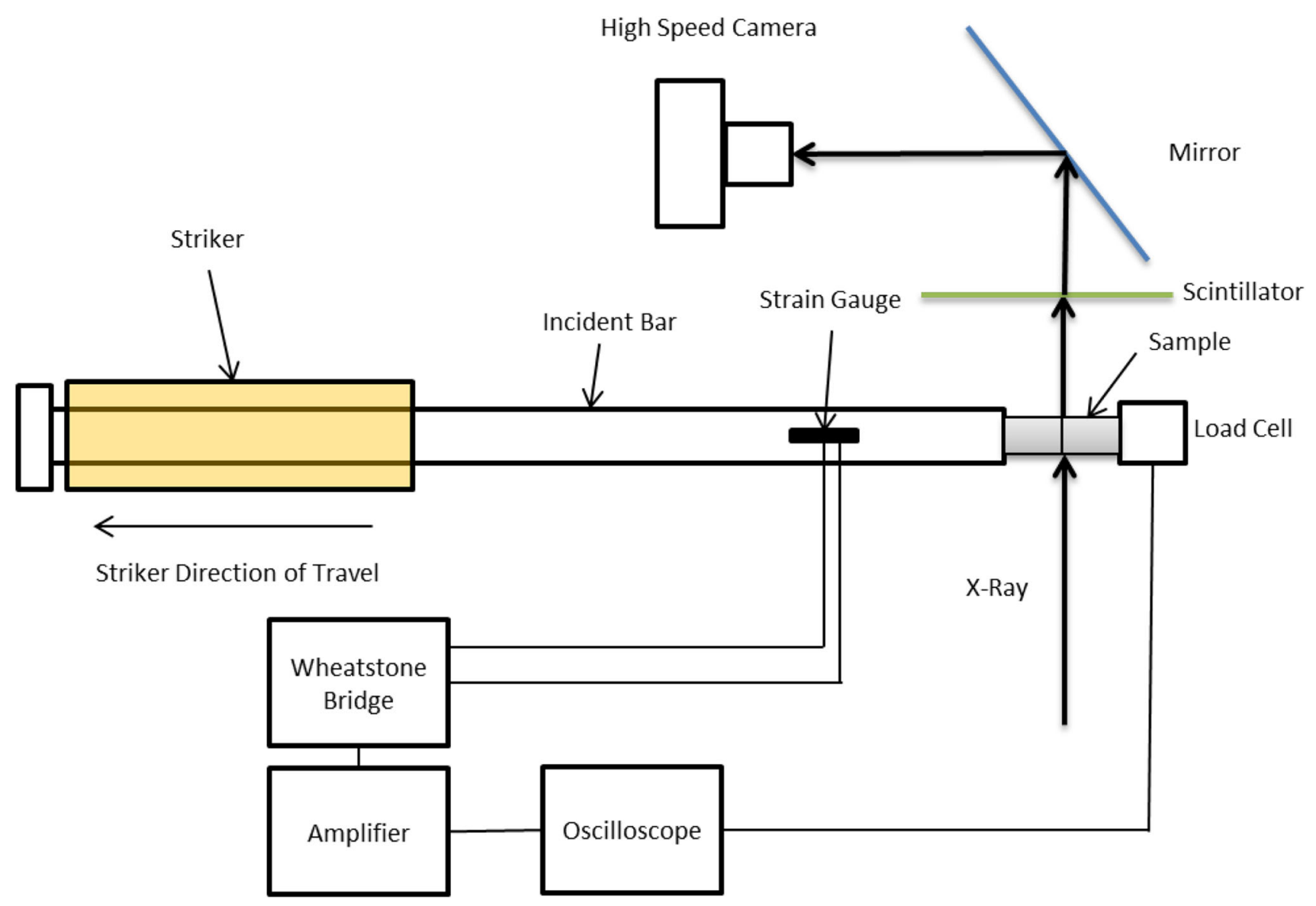

Fig. 5 Experimental schematic detailing wiring to oscilloscope

interface. This point signified the final specimen geometry that was used for experiments and can be seen in Fig. 6. The effectiveness of the specimen geometry was confirmed with a finite element analysis. The analysis showed that keeping the center vertical section of the sample narrow prevented the stress wave from spreading out too much, which in turn increased the likelihood of debonding.

\section{Experimental Results}

\section{Force-Displacement Response}

The transverse dynamic debonding experiments were able to produce viable data that can be used to better understand debonding phenomena. The outputs from the strain gage and the load cell were utilized in order to create a forcedisplacement curve for the debonding events. The samples were loaded at an average velocity of $2.58 \mathrm{~m} / \mathrm{s}$ and an average strain rate of $803 \mathrm{~s}^{-1}$. The high speed imagery confirmed that the primary failure mode was debonding as well as that debonding did initiate away from the free surfaces of the samples. In addition, the images from the high speed camera were synchronized with the force and displacement data to provide further insight into the debonding process.

The force-time curve can be seen in Fig. 7. The entirety of the loading history took place over approximately $45 \mu$ s. This meant that the load cell was able to capture 2250 data points during the loading of the sample. The sample was noted to completely debond during the loading from the initial stress wave. The force displacement curves were generated using the data reduction methods discussed in the "Data Reduction" section. A smoothing function was used for the force output in the force-displacement graph order to reduce the electrical noise present in the data. The beginning and end of the incident and reflected waves were then determined by manually choosing points on the bar strain histories plot. The incident and reflected waves were overlapped in order to determine the sample front-end velocity and displacement. Given that the outputs from the strain gage and load cell used the same universal time frame, the data was then aligned to create the force-displacement curve, as shown in Fig. 8. 


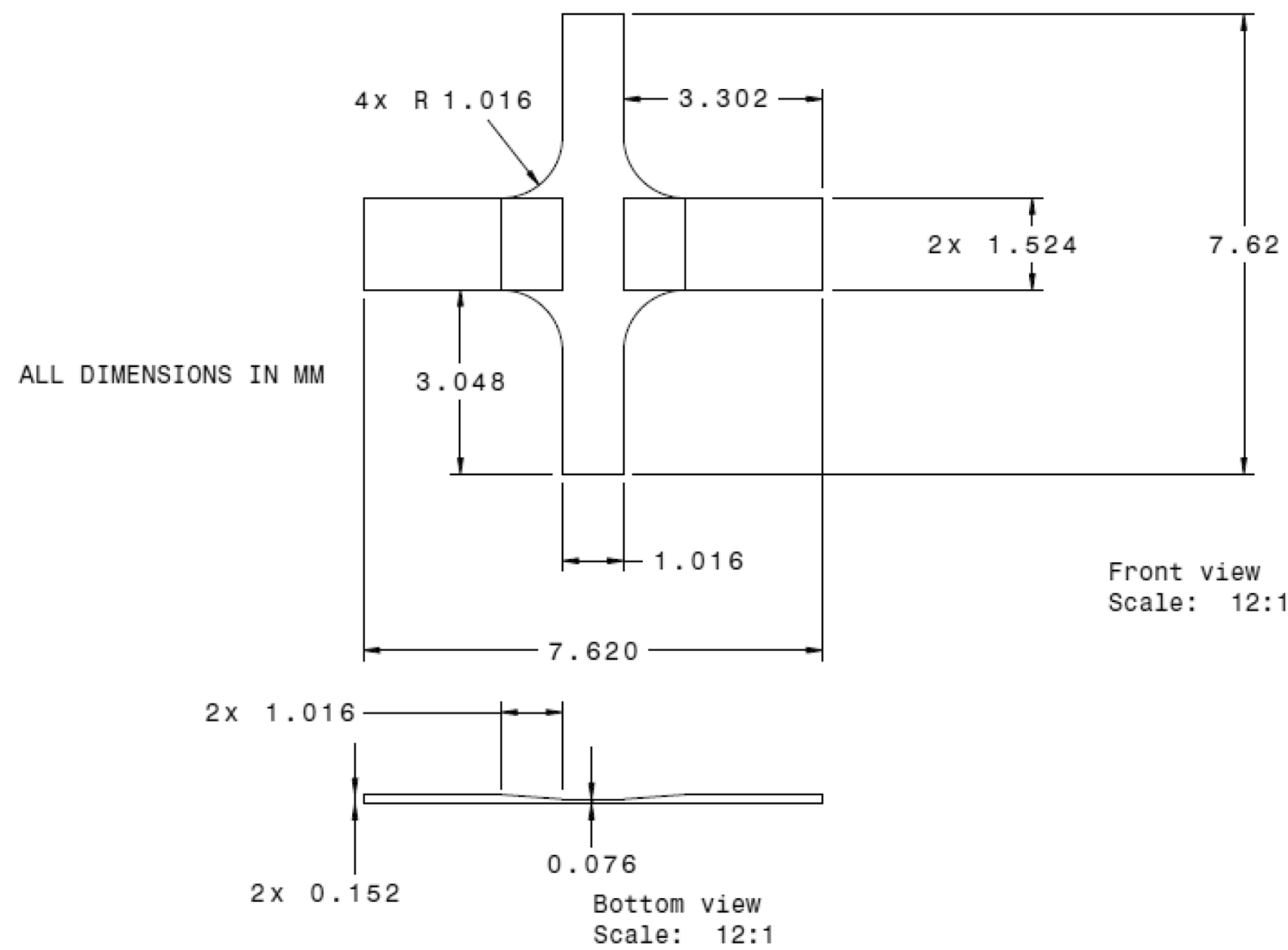

Fig. 6 Final cruciform geometry

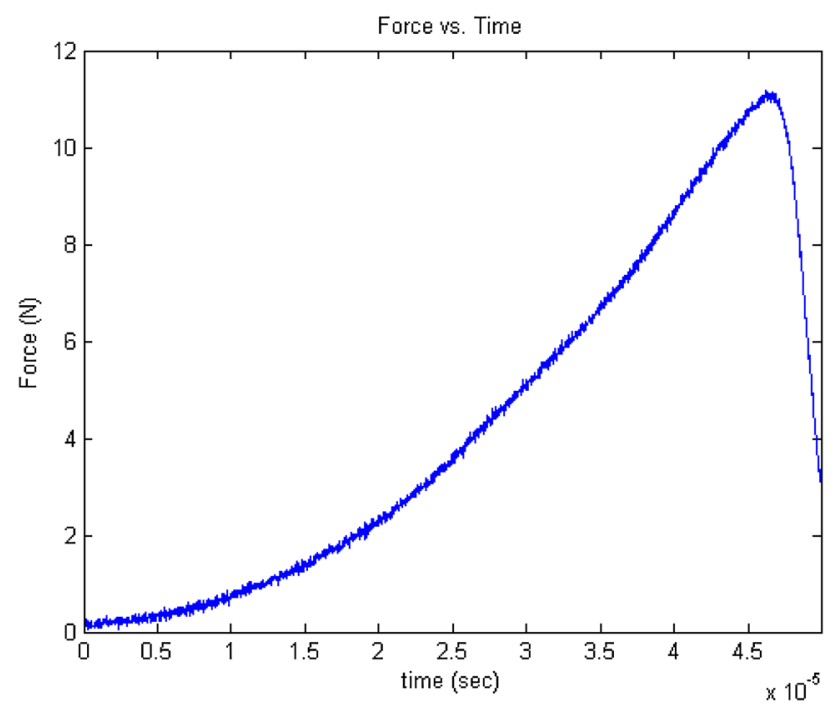

Fig. 7 Force-time response of a sample

The sample thickness in the fiber/matrix interface region as well as the maximum debonding load for each of the samples tested under dynamic loading conditions in shown in Fig. 9. The interfacial stresses can then be calculated by creating analytical models for each loading condition.

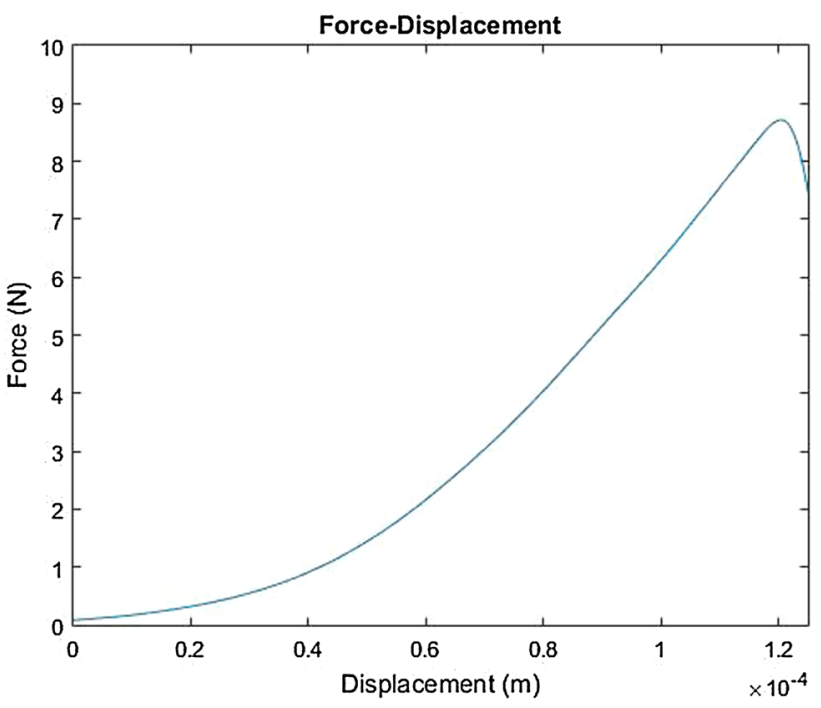

Fig. 8 Force-displacement response of a sample

\section{High Speed Imaging Synchronization}

Once the data reduction was completed, it was of benefit to align the data with the images taken by the high speed camera. In particular, the interface load during each image was able to be determined. The amount of time it took for 
Fig. 9 Debonding load vs. sample thickness for dynamically loaded samples

Fig. 10 Debonding progression

Debonding Stress vs. Sample Thickness

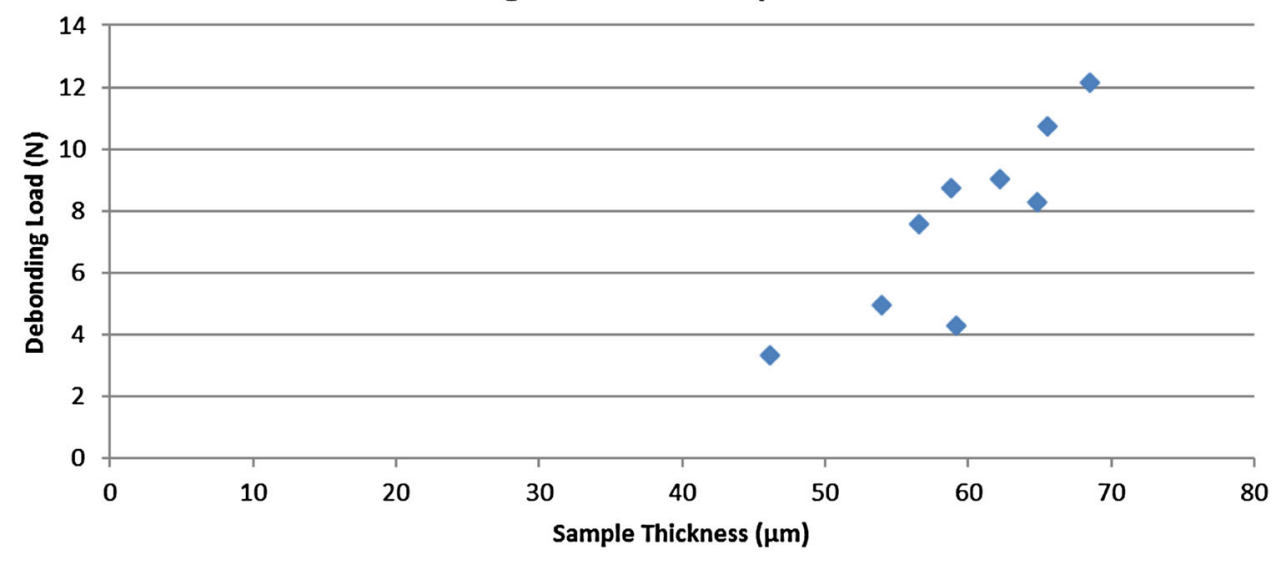

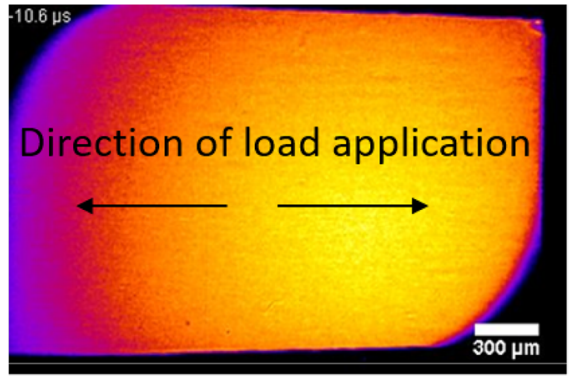

(a) $F=7.15 \mathrm{~N}$

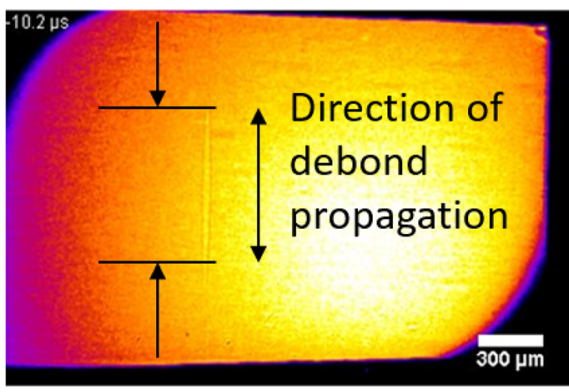

(c) $F=7.28 \mathrm{~N}$

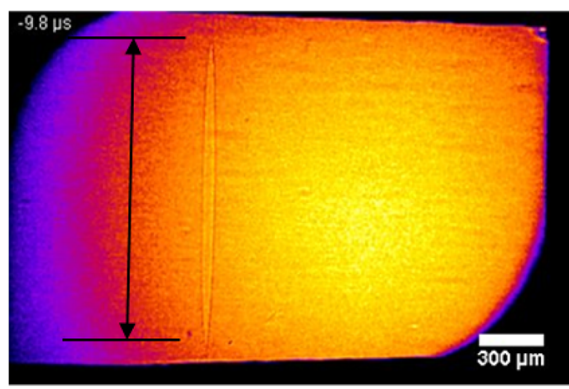

(e) $F=7.41 \mathrm{~N}$

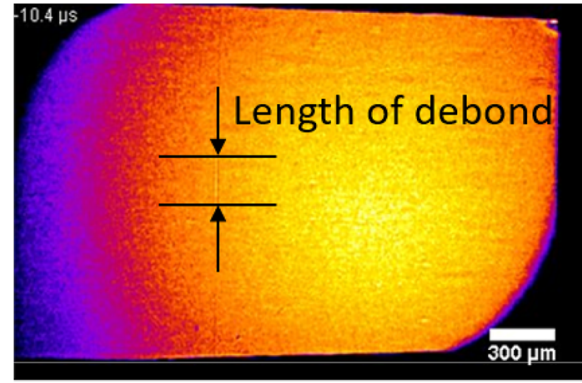

(b) $F=7.24 \mathrm{~N}$

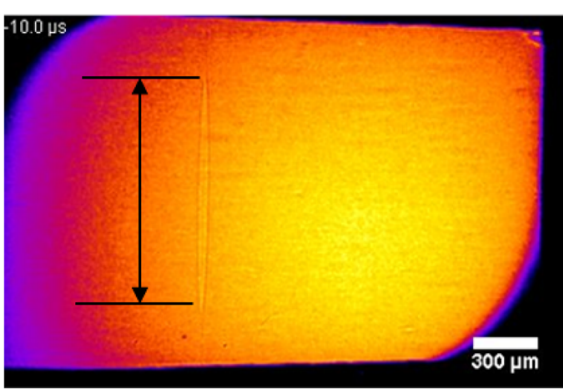

(d) $F=7.34 \mathrm{~N}$

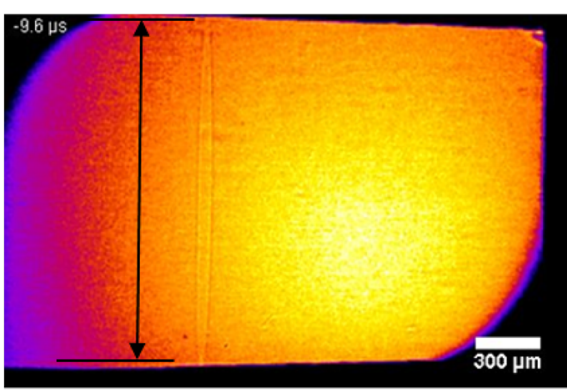

(f) $\mathrm{F}=7.44 \mathrm{~N}$ the wave to travel from the interface to the load cell had to be taken into account in order to properly measure the debonding load. This time was determined by taking half of the sample length and dividing it by the wave velocity of the epoxy resin. This used the assumption that the fiber was approximately located in the center of the sample. Images 


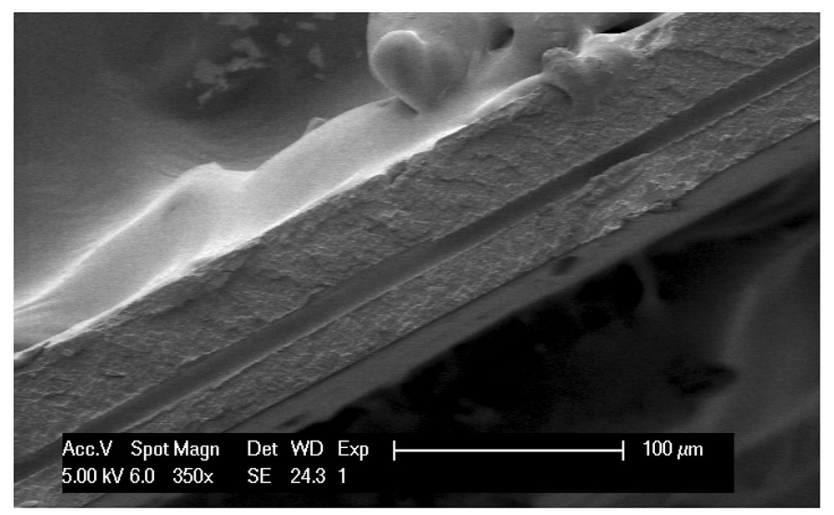

Fig. 11 Failure surface of a sample with indentation from fiber

of the initiation and progression of debonding as well as the interface load for a sample is shown in Fig. 10. Each image in the figure is taken at $200 \mathrm{~ns}$ intervals. The debonding continues after the sixth image, but the X-ray window is only able to record a small area and thus the debonding progresses off screen.

Initially, there is a residual compressive stress at the fiber/matrix interface due to a difference in the coefficient of thermal expansion between the fiber and matrix. As the tensile stress wave reaches the interface, a radial tensile stress is present that changes to a radial compressive stress when progressing circumferentially. These images show Mode I and Mode II loading at the onset of debonding. Mode III loading can be observed as the debond propagates along the fiber length. The crack speed was determined to be at its peak during the initial phases of the debonding process and decreased for each subsequent time step that was captured with the high speed camera, before eventually progressing off screen. The crack speed was determined to be as high as $2200 \mathrm{~m} / \mathrm{s}$, which is approximately equal to the longitudinal wave velocity of the epoxy resin. These images definitively show that by using a proper cruciform design, transverse debonding under dynamic loads initiates away from the free surface. Subsequently, images were taken with an SEM to observe the failure surface. The failure surface for one sample can be seen in Fig. 11. The indentation in the epoxy was the location of the fiber prior to debonding.

Given that materials may behave differently under dynamic and quasi-static loading conditions, it was of interest to compare the maximum debonding load of the samples tested on the Kolsky bar to that of samples tested under quasi-static loading. For the dynamic experiments, 9 samples debonded successfully with an average debonding load of $7.67 \mathrm{~N}$ and a standard deviation of $2.97 \mathrm{~N}$. The samples tested under quasi static loading conditions were loaded at $0.01 \mathrm{~mm} / \mathrm{s}$. Eleven samples were tested, with an average debonding load of $7.31 \mathrm{~N}$ and a standard deviation of $2.86 \mathrm{~N}$, which is a $4.6 \%$ difference in average debonding load. This is small enough that a significant difference in debonding load at different strain rates cannot be inferred. Future experiments with larger data sets and more precisely controlled sample thicknesses may be able to more definitively determine the differences, if any, in the debonding loads.

\section{Conclusions}

Overall, through the iterative manufacturing and testing processes, single fiber transverse dynamic debonding was achieved. Furthermore, by utilizing high speed X-ray imagery at Beamline 32-ID-B at Argonne National Lab's Advanced Photon Source, it was proven that samples with cruciform shaped geometry can debond away from the free edge under dynamic loading conditions. On top of this, the experiments provided insight into the details of debonding loads and progression. This method, combined with X-ray imaging allowed for the ability to visualize debonding initiation and progression. This was accomplished even though the fiber was embedded in an opaque sample. A Kolsky bar was able to provide consistent experimental conditions that made data comparison feasible. The experimental results under dynamic loading conditions were then compared to those obtained under quasi-static loading conditions. These experiments lay the groundwork for further experiments and analytical models that will be able to improve understanding of single fiber debonding under dynamic loading conditions.

Acknowledgments We would like to thank Dr. Kamel Fezzaa, Dr. Tao Sun, and Alex Deriy, for their technical assistance and support of a safe work environment for our experiments at Beamline 32-ID-B. Use of the Advanced Photon Source was supported by the U. S. Department of Energy, Office of Science, Office of Basic Energy Sciences, under Contract No. DE-AC02-06CH11357. The first and last authors' time on this research was sponsored by the Army Research Laboratory and was accomplished under Cooperative Agreement Number W911NF-12-2-0022. The views and conclusions contained in this document are those of the authors and should not be interpreted as representing the official policies, either expressed or implied, of the Army Research Laboratory or the U.S. Government. The U.S. Government is authorized to reproduce and distribute reprints for Government purposes notwithstanding any copyright notation herein.

\section{References}

1. Meurs PF, Schrauwen BA, Schreurs PJ, Peijs T (1998) Determination of the interfacial normal strength using single fibre model composites. Compos Part A 29(9-10):1027-1034

2. Hu S, Karpur P, Matikas TE, Shaw L, Pagano NJ (1995) Free edge effect on residual stresses and debond of a composite fibre/matrix interface. Mech Adv Mater Struct 2:215-225 
3. Pipes RB, Pagano NJ (1970) Composite laminates under uniform axial extension. J Comps Mater 4:538-548

4. Gundel DB, Majumdar BS, Miracle DB (1995) Evaluation of the transverse response of fiber-reinforced composites using a crossshaped sample geometry. Scr Metall Mater 33(12):2057-2065

5. Ageorges C, Friedrich K, Schüller T, Lauke B (1999) Single-fibre Broutman test: fibre-matrix interface transverse debonding. Compos Part A Appl Sci Manuf 30:1423-1434

6. Schüller T, Beckert W, Lauke B, Friedrich K (2000) Single-fibre transverse debonding: tensile test of a necked specimen. Compos Sci Technol 60:2077-2082

7. Ogihara S, Koyanagi J (2010) Investigation of combined stress state failure criterion for glass fiber/epoxy interface by the cruciform specimen method. Compos Sci Technol 70(1):143-150

8. Tandon GP, Kim RY, Bechel VT (2002) Fiber-matrix interfacial failure charcterization using a cruciform-shaped specimen. J Compos Mater 36(23):2667-2691

9. Tandon GP, Kim RY, Bechel VT (2000) Evaluation of interfacial normal strength in a SCS-0/epoxy composite with cruciform specimens. Compos Sci Technol 60:2281-2295

10. Bechel VT, Tandon GP (2002) Characterization of interfacial failure using a reflected light technique. Exp Mech 42(2):200-205
11. Li Z, Ghosh S, Getinet N, O’Brien DJ (2016) Micromechanical modeling and characterization of damage evolution in glass fiber epoxy matrix composites. Mech Mater 99:37-52

12. Bechel VT, Tandon GP (2002) Modified cruciform test for application to graphite/epoxy composites. Mech Adv Mater Struct 9(1):1-17

13. Martyniuk K, Sørensen BF, Modregger P, Lauridsen EM (2013) 3D in situ observations of glass fibre/matrix interfacial debonding. Compos Part A Appl Sci Manuf 55:63-73

14. Chen W, Song B (2010) Split Hopkinson (Kolsky) bar: design, testing and applications. Springer, Berlin

15. Tamrakar S, Haque BZ, Gillespie JW (2016) High rate test method for fiber-matrix interface characterization. Polym Test 52:174-183

16. Li Z, Bi X, Lambros J, Geubelle PH (2002) Dynamic fiber debonding and frictional push-out in mode composite systems: experimental observations. Exp Mech 42(4):417-425

17. Hudspeth M, Claus B, Dubelman S, Black J, Mondal A, Parab N, Funnell C, Hai F, Qi ML, Fezzaa K, Luo SN, Chen W (2013) High speed synchrotron $\mathrm{X}$-ray phase contrast imaging of dynamic material response to split Hopkinson bar loading. Rev Sci Instrum 84(2):025102 